\title{
Variação anual da umidade de equilíbrio e da cor de madeiras amazônicas submetidas a ensaios de campo
}

\author{
Adilson Pacheco de Souza ${ }^{1 \star}$, Luan Lima Mota, Bruno Henrique Casavecchia ${ }^{1}$, Diego Martins Stangerlin ${ }^{1}$, \\ Rafael Rodolfo de Melo \\ ${ }^{1}$ Instituto de Ciências Agrárias e Ambientais, Universidade Federal de Mato Grosso (UFMT), Sinop, Mato Grosso, Brasil. \\ ${ }^{2}$ Universidade Federal do Semi-Árido (UFERSA), Mossoró, Rio Grande do Norte, Brasil.
}

\begin{abstract}
RESUMO Caracterizou-se o teor de umidade de equilíbrio (TUE) e a colorimetria da madeira de dez espécies da região amazônica, submetidas a ensaios de campo (céu aberto), na região Médio-Norte de Mato Grosso, durante um ano (com estação seca de maio a setembro, e chuvosa de outubro a abril). Corpos de prova com as dimensões de $2 \times 2 \times 10 \mathrm{~cm}$ (espessura $\mathrm{x}$ largura $\mathrm{x}$ comprimento), foram dispostos sobre uma plataforma metálica, inclinados a $12^{\circ}$ com face ao Norte. Mensalmente, corpos de prova foram coletados para avaliação do TUE (com base nas relações entre peso úmido e peso seco), enquanto que para a caracterização da cor, empregou-se o sistema CIELAB, com a determinação dos parâmetros $\mathrm{L}^{*}$ (luminosidade), $\mathrm{a}^{\star}$ (coordenada verde-vermelho), $\mathrm{b}^{\star}$ (coordenada amarelo-azul), $\mathrm{C}^{\star}$ (cromaticidade), h (ângulo de tinta) e $\bullet \mathrm{E}$ (variação total da cor). As médias mensais de TUE não apresentaram diferenças entre as espécies, todavia, foram significativas quando agrupadas em classes de densidade básica. Na estação seca, foram observados menores níveis de TUE, independentemente da espécie, em função da redução da umidade relativa do ar. Foram observadas reduções da $L^{*}, C^{*}, a^{*}$ e b* após o período de exposição ao ambiente. As madeiras de amescla e cedro apresentaram o maior e menor valor de $\bullet$ E, decorrentes da baixa densidade básica e dos baixos valores dos parâmetros colorimétricos iniciais (antes da exposição), respectivamente.
\end{abstract}

Palavras-chave: colorimetria; qualidade da madeira; propriedades visuais da madeira; sistema CIEL*a*b.

\section{Annual variation of the equilibrium moisture content and the color of Amazonian woods submitted to field trials}

\begin{abstract}
The equilibrium moisture content (EMC) and the wood color of ten species of the Amazon region was submitted to fiel trials (open sky), in the Mid-North of Mato Grosso State, Brazil, during one year (dry season from May to September; rainy season from October to April). The samples with dimensions of $2 \times 2 \times 10 \mathrm{~cm}$ (thickness, width and length) were placed on a metal platform, inclined to $12^{\circ}$ to face the North. Monthly, samples were collected for evaluation of EMC (based on the relationships between wet weight and dry weight), while for color characterization, The CIELa ${ }^{\star} b^{*}$ system was used to define the parameters $\mathrm{L}^{\star}$ (brightness), $\mathrm{a}^{\star}$ (green-red coordinate), $\mathrm{b}^{\star}$ (yellow-blue coordinate), $\mathrm{C}^{\star}$ (chromaticity), $\mathrm{h}$ (ink angle) and $\bullet \mathrm{E}$ (total color change). The EMC monthly averages showed no differences between species, however, were significant when grouped in classes of basic density. The lower levels of EMC were observed on the dry season, independent of the species, due to the reduction of the relative humidity of the air. Reductions of $\mathrm{L}^{*}, \mathrm{C}^{*}, \mathrm{a}^{*}$ and $\mathrm{b}^{*}$ were observed after the period of exposure to the environment. The woods of amescla and cedro had the highest and lowest value of $\bullet E$, due to the low basic density and the low values of the initial colorimetric parameters (before exposure), respectively.
\end{abstract}

Keywords: colorimetry; quality of wood; visual properties of wood; system CIELa* ${ }^{*}$. 


\section{Introdução}

A madeira pode ser considerada como um material que possui boas características químicas, físicas e mecânicas, que aliado ao alto valor decorativo propiciam grande apresso no seu uso quando comparado com outros materiais, principalmente para aplicações estéticas, de engenharia e estruturais (OZYHAR et al., 2012). Dentre as características interessantes da madeira, podem ser destacadas a alta resistência específica, isolamento térmico, elétrico e acústico, baixo consumo energético no seu processamento, fácil trabalhabilidade manual ou por máquinas (RIBEIRO et al., 2019).

Segundo Silva et al. (2017) para algumas aplicações de madeiras nativas, são mais atrativos os seus atributos estéticos, dados por algumas de suas propriedades organolépticas (propriedades sensoriais), como cor, brilho e desenho. Todavia, esses usos, em geral, caracterizam-se pela exposição da madeira ao ambiente e demandam o conhecimento da sua capacidade de resistir a diferentes intempéries. Nessas situações de exposição, a deterioração da madeira pode gerar comprometimento das suas propriedades físicas, químicas, mecânicas e estéticas, podendo ser resultantes da ação de agentes xilófagos (insetos, microrganismos e brocas marinhas) e das condições ambientais (exposição à radiação solar, chuva, variações de temperatura e umidade relativa do ar), além de reações químicas diversas (STANGERLIN et al., 2013; RIBEIRO et al., 2014; ROMANINI et al., 2014; QUINTILHAN et al., 2018).

A cor da madeira é originada dos depósitos de substâncias corantes no interior das células que constituem o material lenhoso e/ou pelas suas impregnações nas paredes celulares. A cor está entre as principais propriedades da madeira com percepção pelos sentidos humanos, e apresenta uma demanda crescente de seu conhecimento, visto que se trata de um indicador não-destrutivo das interações entre a madeira e o ambiente de exposição, além de permitir a identificação e classificação das madeiras. Todavia, ressalta-se que as variações de cor (tonalidades) da madeira podem ocorrer entre espécies e entre indivíduos, pois depende de fatores genéticos, ambientais, manejo, processamento e tratamento (SILVA et al., 2017).

Por conseguinte, a cor é dependente da composição química de substâncias presentes no xilema (Mady, 2000) e pode ser alterada pela umidade do ar, temperatura do ar e reações fotoquímicas dos componentes químicos presentes na sua estrutura (ZERBINI, 2008; CASSIANO et al., 2013; SILVA et al., 2017). Esses diferentes fatores ambientais degradam as principais componentes químicas da madeira (lignina, celulose e hemicelulose), e por sua vez, a despolimerização de lignina e celulose pode resultar na redução das propriedades físicas, químicas e biológicas da madeira. Essas alterações nas propriedades da madeira são indicadas pela análise dos efeitos do "intemperismo", considerado como um processo dinâmico e lento, dependente das interações das variáveis ambientais e a madeira (BONFATTI JUNIOR; LENGOWSKI, 2018).

Em geral, esses efeitos observados nas alterações da madeira são inicialmente superficiais, podendo atingir até 2,5 $\mathrm{mm}$ abaixo da camada superficial exposta ao ambiente (KERBER et al., 2016). Especificamente para a cor, Hon (2001) e Silva; Pastore (2004) indicam que a radiação solar nos comprimentos de onda do ultravioleta (UV), por apresentarem maiores níveis energéticos, modificam com maior intensidade os componentes químicos dos principais polímeros constituintes da madeira (celulose, lignina, hemicelulose e extrativos). Essa fotodegradação oxida os constituintes químicos, formando radicais livres, modificando a cor da madeira, enquanto que, a água (proveniente das precipitações em ambiente aberto), lixivia os constituintes fotodegradados, expondo uma nova camada da 
madeira ao ambiente (HON, 2001; BONFATTI JUNIOR; LENGOWSKI, 2018).

A madeira é um material higroscópico e de comportamento anisotrópico, que está sujeita a trocas de vapor d'água com a atmosfera (CASSIANO et al., 2013). Essas trocas tendem a se estabilizar ao longo do tempo, atingindo o equilíbrio com a umidade do ar e definindo a umidade de equilíbrio (UE) da madeira, que por sua vez, é um dos fatores que afeta as variações de cor da madeira exposta ao ambiente.

Em função da importância das madeiras amazônicas, das suas identificações e indicações de usos, o presente trabalho contribui na abordagem conjunta entre umidade de equilíbrio e colorimetria da madeira. Desse modo, o presente trabalho tem por objetivo caracterizar a alteração da umidade de equilíbrio (UE) e da estabilidade colorimétrica da madeira de dez espécies comerciais da Amazônia, em ensaio de campo, ao longo do ano, na região Médio-Norte do Mato Grosso.

\section{Material e Métodos}

O experimento foi conduzido na área da estação meteorológica do Campus Universitário de Sinop, da Universidade Federal de Mato Grosso, localizada nas coordenadas $11,85^{\circ} \mathrm{S}$ e $55,49^{\circ} \mathrm{W}$, com altitude de $370 \mathrm{~m}$, entre dezembro de 2013 a dezembro de 2014. A região apresenta clima classificado como Aw (classificação de Koopen), com duas estações hídricas bem definidas: seca (maio a setembro) e chuvosa (outubro a abril); a temperatura média anual é de $24,7{ }^{\circ} \mathrm{C}$, com médias mensais entre 23,0 e $25,8{ }^{\circ} \mathrm{C}$; e precipitações em torno de $1974 \mathrm{~mm}$ ano $^{-1}$ (SOUZA et al., 2013).

Foram obtidas, em diferentes madeireiras do município de Sinop - Mato Grosso, tábuas de madeiras de dez espécies amazônicas: Amescla "Trattinickia burserifolia Mart." (Burseraceae), Angelim pedra "Dinizia excelsia Ducke"
(Fabaceae/ Mimosoideae), Cambará “Qualea paraensis Ducke” (Vochysiaceae), Canelão “Ocotea velutina Mart.” (Lauraceae), Cedro "Erisma uncinatum Warm." (Vochysiaceae), Cumaru "Dipteryx odorata (Aubl.) Willd." (Fabaceae), Guanandi "Calophyllum brasiliense Camb." (Clusiaceae), Garapeira "Apuleia leiocarpa Vog. Macbride” (Fabaceae/ Caesalpiniaceae), Itaúba “Mezilaurus itauba Meisn." (Lauraceae) e Jatobá "Hymenaea courbaril L." (Fabaceae/ Caesalpiniaceae).

As madeiras foram beneficiadas em plaina desempenadeira-desengrossadeira e seccionadas em serra circular de mesa, para confecção dos corpos de prova nas dimensões de $2 \times 2 \times 10 \mathrm{~cm}$, com a maior dimensão na direção longitudinal. Foram selecionados 10 corpos de prova para determinação da densidade básica (db), conforme procedimento descritos na NBR 7190 (ABNT, 1997). A umidade de equilíbrio inicial da madeira (Tabela 1) foi obtida com base em peso seco, em estufa de circulação forçada de ar a $103{ }^{\circ} \mathrm{C}$ ) até a obtenção de massa constante, com massa úmida e massa seca obtidas em balança eletrônica digital (precisão de $\pm 0,01 \mathrm{~g}$ ). Visando realizar análises agrupadas de espécies, dividiu-se as dez espécies em três grupos de densidade básica, conforme a classificação proposta por Melo et al. (1990), que estabelece as classes baixa $\left(\mathrm{db}<0,5 \mathrm{~g} \mathrm{~cm}^{-3}\right)$, média $\left(0,51<\mathrm{db}<0,5 \mathrm{~g} \mathrm{~cm}^{-3}\right)$ e alta $\left(\mathrm{db}>0,71 \mathrm{~g} \mathrm{~cm}^{-3}\right)$.

Para o ensaio de intemperismo natural foram selecionados 60 corpos de prova por espécie. Estes foram dispostos em uma plataforma metálica com inclinação igual a latitude local e face para o Norte, apoiados sobre uma tela metálica de malha de $1 \mathrm{~cm}^{2}$, permitindo assim a circulação de ar (CASSIANO et al., 2013). O arranjo experimental foi fatorial 10 x 12 (espécies x meses), com cinco repetições dadas pelo agrupamento dos corpos de prova em blocos casualizados 
As coletas dos corpos de prova foram realizadas mensalmente, durante um ano, entre as 13:00 e 15:00 horas em função da maior predominância dos menores valores diários de umidade relativa do ar (para a região), independente da época do ano (SANTOS et al., 2013). Após serem retirados do ambiente, os corpos de provas foram pesados em balança eletrônica digital (precisão de $\pm 0,01 \mathrm{~g}$ ) para obtenção da massa úmida, e submetidos à secagem em estufa de circulação forçada de $\operatorname{ar}\left(103^{\circ} \mathrm{C}\right)$ até a obtenção de massa constante.

Tabela 1. Caracterização inicial dos corpos de prova de dez espécies de madeira amazônica submetidas ao intemperismo natural.

Table 1. Characterization of ten Amazonian wood samples subjected to natural weathering.

\begin{tabular}{ccccc}
\hline Cod. & Nome & $\begin{array}{c}\text { Densidade } \\
\text { básica }\left(\mathbf{g ~ c m}^{-3}\right)\end{array}$ & $\begin{array}{c}\text { Classe de } \\
\text { Densidade }\end{array}$ & $\begin{array}{c}\text { Umidade } \\
\text { inicial }\end{array}$ \\
\hline AM & Amescla & $0,49 \mathrm{f}$ & Baixa & $12,52 \mathrm{a}$ \\
AG & Angelim & $0,59 \mathrm{e}$ & Média & $10,01 \mathrm{c}$ \\
CB & Cambará & $0,65 \mathrm{~d}$ & Média & $11,31 \mathrm{~b}$ \\
CN & Canelão & $0,64 \mathrm{~d}$ & Média & $7,59 \mathrm{e}$ \\
CD & Cedro & $0,56 \mathrm{e}$ & Média & $9,57 \mathrm{c}$ \\
CU & Cumaru & $0,92 \mathrm{a}$ & Alta & $6,85 \mathrm{f}$ \\
GD & Guanandi & $0,75 \mathrm{c}$ & Alta & $10,02 \mathrm{c}$ \\
GP & Garapeira & $0,86 \mathrm{~b}$ & Alta & $6,34 \mathrm{f}$ \\
IT & Itaúba & $0,77 \mathrm{c}$ & Alta & $10,52 \mathrm{c}$ \\
JB & Jatobá & $0,86 \mathrm{~b}$ & Alta & $8,79 \mathrm{~d}$ \\
\hline
\end{tabular}

Cod. = Código; Médias seguidas da mesma letra na coluna não diferem entre si a 5\% de probabilidade pelo teste de ScottKnott.

Após secagem, os corpos de prova foram pesados novamente para obtenção de massa seca, sendo posteriormente, determinado o teor de umidade de equilíbrio (TUE).

$$
\mathrm{TUE}=[(\mathrm{Mu}-\mathrm{Ms}) / \mathrm{Ms}] \times 100
$$

Em que: TUE $=$ teor de umidade de equilíbrio; $\mathrm{Mu}=$ massa úmida (g); Ms = massa seca $(\mathrm{g})$.
Durante o período experimental, o monitoramento meteorológico ocorreu em uma estação automática, com o sistema de aquisição de dados CR 1000 da Campbell Scientific, com sensores de radiação solar global piranômetro (CS300), psicrômetro com abrigo termométrico (Vaisala, CS 215), ambos a $2 \mathrm{~m}$ de altura e pluviógrafo (TE 525) a 1,5 m de altura.

A caracterização colorimétrica foi realizada no plano tangencial da madeira por meio de um colorímetro com resolução de 3nm de iluminação difusa, iluminante D65 composto por lâmpada de xenônio com ângulo de observação $10^{\circ}$ e área de iluminação de $11 \mathrm{~mm}$ de diâmetro. Antecedendo as análises, realizou-se a calibração do aparelho com auxílio de uma amostra referência fornecido junto ao equipamento, com as seguintes características colorimétricas: $Y=86,8 ; x=$ 0,$3193 ; y=0,3365$.

Os parâmetros colorimétricos $\mathrm{L}^{\star}$ (luminosidade), $\mathrm{a}^{*}$ (coordenada verde-vermelho), $\mathrm{b}^{*}$ (coordenada azulamarelo), $\mathrm{C}^{\star}$ (cromaticidade) e h (ângulo de tinta) foram obtidos ao empregar o sistema CIEL ${ }^{\star} a^{\star} b^{\star}$ e a coordenada de cor $\mathrm{CIE} \mathrm{L}^{\star} \mathrm{C}^{\star} \mathrm{h}$, sendo realizada a média de três leituras para cada corpo de prova, na face exposta a radiação solar. Ressalta-se que, os corpos de prova foram seccionados a 5,0 $\mathrm{cm}$ no sentido longitudinal, para análises de cor e de umidade separadamente. Adicionalmente, determinou-se a variação total da cor $\left(\bullet E^{\star} a b\right)$ (Equação 2), conforme a padronização D 2244 (ASTM, 2009).

$$
\Delta \mathrm{E} * \mathrm{ab}=\sqrt{\Delta \mathrm{L}^{* 2}+\Delta \mathrm{a}^{*^{2}}+\Delta \mathrm{b}^{* 2}}
$$

Em que: $\bullet E=$ variação total da cor; $\bullet L^{*}=$ diferença da leitura da luminosidade antes e após o intemperismo; $\cdot \mathrm{a}^{*}=$ diferença da leitura da coordenada verde-vermelho antes e após o intemperismo; $\cdot \mathrm{b}^{*}=$ diferença da leitura da coordenada azul-amarelo antes e após o intemperismo.

As médias de umidade de equilíbrio e variação total da cor foram submetidas a análise de variância, pelo teste $\mathrm{F}$, e 
comparadas pelo teste de Tukey, a 5\% de probabilidade de erro. Adicionalmente, realizou-se a análise de componentes principais (ACP) pelo software R, que permite condensar a informação original contida nos parâmetros colorimétricos $\left(L^{\star}, a^{\star}, b^{*}, C^{\star} e \mathrm{~h}\right)$, em duas variáveis ortogonais, que são combinações lineares das variáveis originais criadas com os dois maiores autovalores da matriz de covariância dos dados (Hair Junior et al., 2009).

Portanto, o conjunto inicial de 5 parâmetros colorimétricos passou a ser caracterizado por duas novas variáveis latentes, possibilitando assim sua localização em figuras bidimensionais (ordenação dos acessos por componentes principais). A adequação desta análise é verificada pela quantidade de informação total das variáveis originais retida pelos componentes principais que mostram autovalores superiores à unidade "1,0" (HAIR JUNIOR et al., 2009). Autovalores inferiores à unidade não dispõem de informação relevante. Assim, os parâmetros colorimétricos das madeiras analisados com a ACP são agrupados de acordo com suas semelhanças e foram separadas por critérios de dissimilaridade, o que levou à formação de grupos, podendo ser validada ou não a utilização da ACP para esse fim.

\section{Resultados e Discussão}

O comportamento do regime pluvial da região indica a formação de duas estações distintas (Figura 1), afetando a temperatura do ar (Tar), a umidade relativa do ar (UR) e a radiação solar global, corroborando com Souza et al. (2013). No período seco (maio a setembro) as amplitudes térmicas foram crescentes, com variações de 13,55 a $17,77^{\circ} \mathrm{C}$ para maio e agosto, respectivamente. Todavia no período chuvoso (outubro a abril) observou-se uma redução da amplitude térmica $\left(8,73^{\circ} \mathrm{C}\right.$ em dezembro), com temperaturas médias diárias entre 24,38 e $25,40^{\circ} \mathrm{C}$.
Segundo Souza et al. (2013), na região Norte e Noroeste do estado de Mato Grosso, entre 2008 e 2011, as temperaturas médias diárias oscilaram entre 9,5 e $37,4^{\circ} \mathrm{C}$, com amplitudes térmicas diárias máximas de $20,5^{\circ} \mathrm{C}$. Entre agosto e setembro são verificadas as maiores temperaturas diurnas para essas regiões, visto que ocorrem menores índices de nebulosidade e maior transmissividade atmosférica da radiação, permitindo maior incidência de radiação direta (comprimentos de onda na faixa do ultra-violeta) e fotodegradação da cor da madeira.

Entre maio e setembro, a UR média diária variou de 54,08 a 78,37\% (com mínimas diárias de 32,13; 24,84 e 34,89\%, entre julho e setembro, respectivamente), enquanto no período chuvoso, as médias diárias foram superiores a $80 \%$. Quanto às precipitações pluviais, observaram-se totais acumulados de 1798,97 mm no período de avaliação, sendo que destes, 36,8 e $24,8 \%$ foram concentrados em dezembro e fevereiro.

As variações dos elementos meteorológicos influenciaram diretamente no teor de umidade de equilíbrio da madeira exposta ao ambiente (ensaios de campo) durante o período de avaliação (07/12/2013 e 06/12/2014). Foram observadas diferenças significativas de TUE na interação entre espécies $x$ tempo de exposição (coleta) apenas para a madeira de amescla, sendo esse comportamento associado a elevada diferença mínima significativa (DMS) obtido para diferenciação entre os valores médios. Todavia, quando analisadas em grupos dependentes da densidade básica $(\mathrm{Db})$ ocorreram diferenças significativas, principalmente entre as estações chuvosa (outubro a abril) e seca (maio a setembro) (Tabela 2). Esse comportamento pode ser justificado em função da baixa densidade da madeira de amescla, que indica maior disponibilidade de espaços para trocas de vapor d'água entre a madeira e o ar atmosférico. 

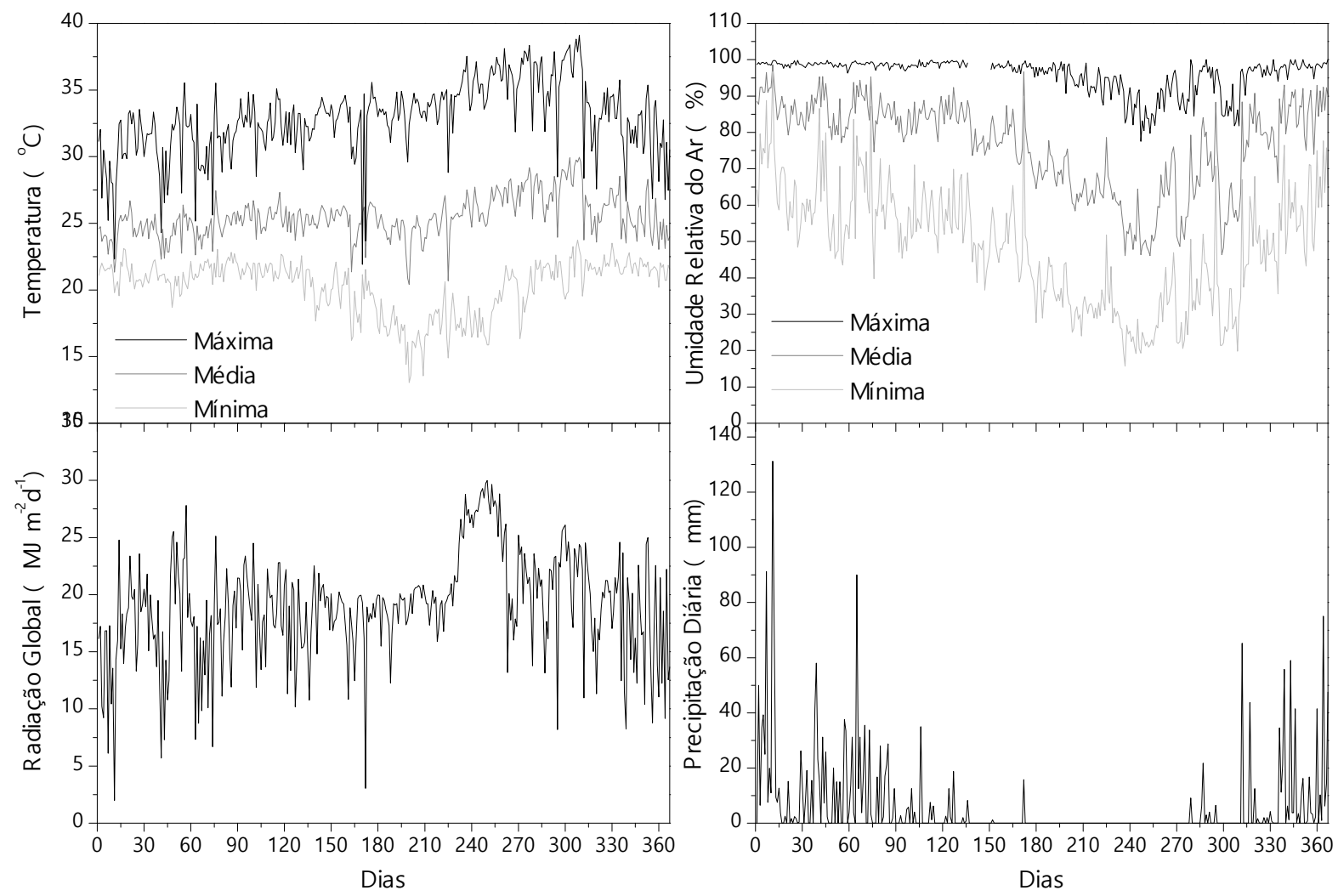

Figura 1. Variações diárias da temperatura do ar, umidade relativa do ar, radiação global e precipitação, entre 07/12/2013 e 06/12/2014, na região de município de Sinop (MT).

Figure 1. Daily variation of air temperature, relative air humidity, global radiation and precipitation between $07^{\text {th }}$ December 2013 and $06^{\text {th }}$ December 2014 in the region of Sinop City (MT).

Tabela 2. Análise fatorial do teor de umidade de equilíbrio das madeiras de 10 espécies amazônicas em diferentes épocas de coleta (meses) submetidas ao intemperismo natural.

Table 2. Factorial analysis of equilibrium moisture content of wood from ten Amazonian species subjected to natural weathering considering different sampling periods (months).

\begin{tabular}{|c|c|c|c|c|c|}
\hline FV & GL & SQ & QM & Fc & $\operatorname{Pr}>\mathrm{Fc}$ \\
\hline \multicolumn{6}{|c|}{ "Espécie x Tempo" } \\
\hline Espécie & 9 & 9111,02 & 1012,34 & 39,96 & $0,0000^{*}$ \\
\hline Coleta & 11 & 4812,51 & 437,50 & 17,27 & $0,0000^{*}$ \\
\hline Espécie x Coleta & 99 & 2702,69 & 27,30 & 1,08 & $0,3030^{\mathrm{NS}}$ \\
\hline Erro & 480 & 12161,58 & 25,34 & & \\
\hline Total corrigido & 599 & 28787,80 & & & \\
\hline \multicolumn{6}{|c|}{ "Densidade básica (Db) x Tempo } \\
\hline $\mathrm{Db}$ & 2 & 8180,05 & 4090,03 & 668,92 & $0,0000^{\star}$ \\
\hline Coleta & 11 & 4530,71 & 411,88 & 67,36 & $0,0000^{*}$ \\
\hline Db x Coleta & 22 & 452,41 & 20,56 & 3,36 & $0,0000^{*}$ \\
\hline Erro & 564 & 3448,48 & 6,11 & & \\
\hline Total corrigido & 599 & 16611,65 & & & \\
\hline
\end{tabular}

Em que: $\mathrm{FV}$ = fonte de variação; $\mathrm{GL}$ = graus de liberdade; $\mathrm{SQ}=$ soma dos quadrados; $\mathrm{QM}=$ quadrado médio; $\mathrm{FC}$ = valor de $\mathrm{F}$ calculado; $\operatorname{Pr}=$ probabilidade; ${ }^{*}=$ significativo a $5 \%$ de probabilidade; NS = não significativo. 
Para a madeira de Amescla foram obtidas variações de TUE entre 19,35 e $25,42 \%$ para agosto e janeiro, respectivamente, sendo que entre as estações chuvosa e seca ocorrem diferenças de 2,33\% (Tabela 3). Independentemente da espécie, observou-se uma redução de TUE em novembro. Segundo Souza et al. (2013), em outubro ocorrem precipitações acumuladas de 182,3 $\mathrm{mm}$ na região de Sinop, todavia, no ano de 2014, foram obtidos apenas $127,7 \mathrm{~mm}$, sendo que destes, 90,4 $\mathrm{mm}$ foram concentrados em apenas 5 dias (terceiro decêndio do mês), que por sua vez, foram antecedentes a 11 dias da coleta realizada em novembro (Figura 1).

Em geral, nas estações secas ocorrem menores valores médios de TUE quando comparado com a estação chuvosa. Dentre as madeiras de média e alta densidade básica, foram obtidas diferenças entre TUE máximo e mínimo de 12,72 e
$11,56 \%$ para cambará e cedro, e de 7,25 e 7,85\% para garapeira e cumaru. Cassiano et al. (2013) avaliaram as madeiras de Angelim Pedra, Canelão, Garapeira, Guanandi e Jatobá expostas ao ambiente no início da estação seca na região de Sinop e observaram valores semelhantes aos obtidos nesse trabalho.

Segundo Martins et al. (2003), pela aplicação do modelo de Simpson (1971), na região Norte e parte da região litorânea brasileira, as médias anuais de TUE variaram de 16 a 18\%, enquanto que para o estado de Mato Grosso, as oscilações ocorreram entre 14 e 16\% nas médias anuais de uma normal climatológica de 1931 a 1990. Nesse contexto, esses níveis de TUE obtidos por esse método, corroboraram apenas na estação chuvosa (outubro a abril) e para madeiras de média e alta densidade básica.

Tabela 3. Variação média mensal do teor de umidade de equilíbrio (em porcentagem) das madeiras de 10 espécies amazônicas submetidas ao intemperismo natural.

Table 3. Monthly average variation of equilibrium moisture content (in percentage) of ten Amazonian wood species subjected to natural weathering.

\begin{tabular}{|c|c|c|c|c|c|c|c|c|c|c|}
\hline Época do ano & Amescla & Angelim Pedra & Cambará & Canelão & Cedro & Cumaru & Guanandi & Garapeira & Itaúba & Jatobá \\
\hline Jan & $25,42^{*}$ & 12,60 & 16,01 & 14,00 & 15,34 & 10,76 & 14,08 & 12,12 & 13,87 & 12,11 \\
\hline Fev & $24,57^{\star}$ & 11,69 & 13,21 & 13,36 & 13,97 & 10,55 & 12,93 & 11,11 & 17,18 & 11,39 \\
\hline Mar & $25,22^{*}$ & 15,34 & 18,78 & 16,56 & 19,41 & 13,08 & 16,66 & 13,60 & 16,49 & 14,96 \\
\hline Abr & $22,44^{\star}$ & 11,36 & 12,09 & 12,30 & 13,56 & 10,03 & 12,63 & 10,71 & 10,13 & 6,53 \\
\hline Mai & $20,50^{*}$ & 8,97 & 8,67 & 11,64 & 10,89 & 9,23 & 10,29 & 9,91 & 9,67 & 9,72 \\
\hline Jun & $22,40^{*}$ & 9,22 & 10,18 & 10,81 & 11,51 & 8,49 & 10,26 & 9,77 & 10,06 & 9,77 \\
\hline Jul & $21,49^{*}$ & 6,16 & 6,57 & 8,40 & 8,20 & 6,77 & 7,15 & 7,12 & 8,41 & 7,18 \\
\hline Ago & $19,35^{*}$ & 6,56 & 6,78 & 8,05 & 8,77 & 6,43 & 7,58 & 7,11 & 7,91 & 6,84 \\
\hline Set & $21,62^{*}$ & 6,67 & 7,40 & 6,57 & 8,33 & 5,23 & 7,50 & 6,53 & 6,48 & 6,79 \\
\hline Out & $20,84^{*}$ & 8,89 & 9,89 & 9,96 & 10,97 & 8,79 & 10,17 & 8,71 & 9,74 & 9,38 \\
\hline Nov & $20,64^{*}$ & 5,79 & 6,06 & 7,73 & 7,85 & 6,60 & 7,05 & 6,35 & 7,85 & 6,95 \\
\hline Dez & $24,67^{\star}$ & 14,65 & 16,80 & 11,17 & 18,62 & 7,54 & 14,79 & 10,29 & 9,35 & 11,75 \\
\hline Média anual & 22,43 & 9,83 & 11,04 & 10,88 & 12,29 & 8,63 & 10,92 & 9,44 & 10,60 & 9,45 \\
\hline Média seca & 21,07 & 7,52 & 7,92 & 9,09 & 9,54 & 7,23 & 8,56 & 8,09 & 8,51 & 8,06 \\
\hline Média chuvosa & 23,40 & 11,47 & 13,26 & 12,15 & 14,25 & 9,62 & 12,62 & 10,41 & 12,09 & 10,44 \\
\hline
\end{tabular}

* Médias da madeira de Amescla diferem das médias das demais espécies para o mesmo mês, a $5 \%$ de probabilidade pelo teste de Scott-Knott.

Os valores de TUE podem ser influenciados pela associação entre a densidade básica e a estação do ano, visto que, mesmo no período seco (maio a setembro), madeira com menor $\mathrm{Db}$ apresentou maior TUE, todavia sem diferenças significativas para madeiras de média e alta $\mathrm{Db}$ (Tabela 4). Segundo Glass et al. (2010) e Silveira et al. (2013), o teor de umidade é inversamente proporcional à densidade da madeira, ou seja, quanto maior a quantidade de água, menor 
a quantidade dos outros elementos químicos da madeira (celulose, hemicelulose, lignina e extrativos).

Aliado a isso, apesar de não ter sido realizada a caracterização química das madeiras, segundo Jankowsky; Galvão (1979) e Kilic; Niemz (2012), espécies com alto teor de extrativos tendem a apresentam maior estabilidade dimensional, menor umidade no ponto de saturação das fibras e umidade de equilíbrio mais baixas do que espécies que possuem menores concentrações de extrativos.

Assim, as variações de TUE podem estar relacionadas com a presença/deterioração da lignina e dos extrativos fenólicos (constituintes químicos menos hidrofílicos), pois com as suas oxidações, podem ocorrer maior absorção de água na madeira em função do aumento da concentração de carboidratos (hemiceluloses e celulose) e consequentemente da presença de sítios de hidroxilas disponíveis (ROWEL, 2005).

De modo geral, os valores dos parâmetros colorimétricos das madeiras expostas ao ambiente reduziram em comparação a condição inicial, antes da exposição ao intemperismo natural, com exceção de h que apresentou acréscimo (Figura 2).

O parâmetro $L^{*}$, mesmo tendo uma variação nas análises mensais, apresentou para todas as madeiras tendência de queda dos valores, indicando escurecimento das madeiras. Amescla e Cedro apresentaram a maior e menor amplitude de luminosidade $(30,22$ e 1,51), respectivamente.

Pela redução das coordenadas colorimétricas $a^{*} e b^{*}$ observou-se uma tendência das madeiras se tornarem menos avermelhadas e amareladas, respectivamente, podendo esse comportamento ser atribuído à deterioração e/ou lixiviação de compostos cromóforos presentes nas camadas superficiais das madeiras (SILVA; PASTORE, 2004). Os resultados obtidos para as variações dessas coordenadas $\left(a^{*}\right.$ e $\left.b^{*}\right)$ corroboram com os verificados por Turkoglu et al. (2015) para madeiras de Pinus sylvestris L. e Fagus orientalis L. após 6 meses (180 dias) de exposição ao intemperismo natural. As madeiras com maior e menor alteração de $\mathrm{a}^{\star}$ foram Guanandi e Amescla (com 15,41 e 3,69\%), respectivamente. Já em relação ao parâmetro $b^{\star}$, as madeiras de Itaúba e Cedro $(24,15$ e 13,21) apresentaram as menores variações, respectivamente.

Tabela 4. Variação média mensal do teor de umidade de equilíbrio (em porcentagem) para madeiras de 10 espécies amazônicas em diferentes classes de densidade básica (Db), submetidas ao ensaio de campo.

Table 4. Monthly average variation of equilibrium moisture content (in percentage) of ten Amazonian wood species subjected to natural weathering considering different levels of basic density $(\mathrm{Db})$.

\begin{tabular}{cccc}
\hline Época do Ano & Db Baixa & Db Média & Db Alta \\
\hline Jan & $25,42 \mathrm{Aa}$ & $14,41 \mathrm{Bb}$ & $12,17 \mathrm{Ab}$ \\
$\mathrm{Fev}$ & $24,57 \mathrm{Aa}$ & $13,04 \mathrm{Bb}$ & $12,56 \mathrm{Ab}$ \\
Mar & $25,22 \mathrm{Aa}$ & $17,35 \mathrm{Ab}$ & $14,53 \mathrm{Ac}$ \\
$\mathrm{Abr}$ & $22,44 \mathrm{Ba}$ & $12,39 \mathrm{Cb}$ & $9,35 \mathrm{Bc}$ \\
Mai & $20,50 \mathrm{Ba}$ & $10,09 \mathrm{Cb}$ & $9,64 \mathrm{Bb}$ \\
Jun & $22,40 \mathrm{Ba}$ & $10,30 \mathrm{Cb}$ & $9,52 \mathrm{Bb}$ \\
Jul & $21,49 \mathrm{Ba}$ & $7,40 \mathrm{Db}$ & $7,30 \mathrm{Cb}$ \\
Ago & $19,35 \mathrm{Ba}$ & $7,57 \mathrm{Db}$ & $7,07 \mathrm{Cb}$ \\
Set & $21,62 \mathrm{Ba}$ & $7,29 \mathrm{Db}$ & $6,26 \mathrm{Cb}$ \\
Out & $20,84 \mathrm{Ba}$ & $9,97 \mathrm{Cb}$ & $9,15 \mathrm{Bb}$ \\
Nov & $20,64 \mathrm{Ba}$ & $6,83 \mathrm{Db}$ & $6,94 \mathrm{Cb}$ \\
Dez & $24,67 \mathrm{Aa}$ & $15,21 \mathrm{Bb}$ & $9,71 \mathrm{Bc}$ \\
\hline Média anual & 22,43 & 10,99 & 9,52 \\
Média - seca & 21,07 & 8,53 & 7,96 \\
Média - chuvosa & 23,40 & 12,74 & 10,63 \\
\hline
\end{tabular}

Médias seguidas da mesma letra maiúscula na coluna e minúsculas na linha, não diferem entre si a $5 \%$ de probabilidade pelo teste de Scott-Knott.

A cromaticidade é matematicamente dependente das coordenadas $\mathrm{a}^{\star} \mathrm{e}^{\star}$, portanto, também reduziu ao longo do tempo, indicando menor saturação da cor e consequentemente um aspecto acinzentado na superfície das madeiras. Esse aspecto acinzentado está relacionado a combinação do clareamento (resultante da degradação da lignina) e do escurecimento (resultante da colonização por fungos emboladores) (CADEMARTORI et al., 2015). 


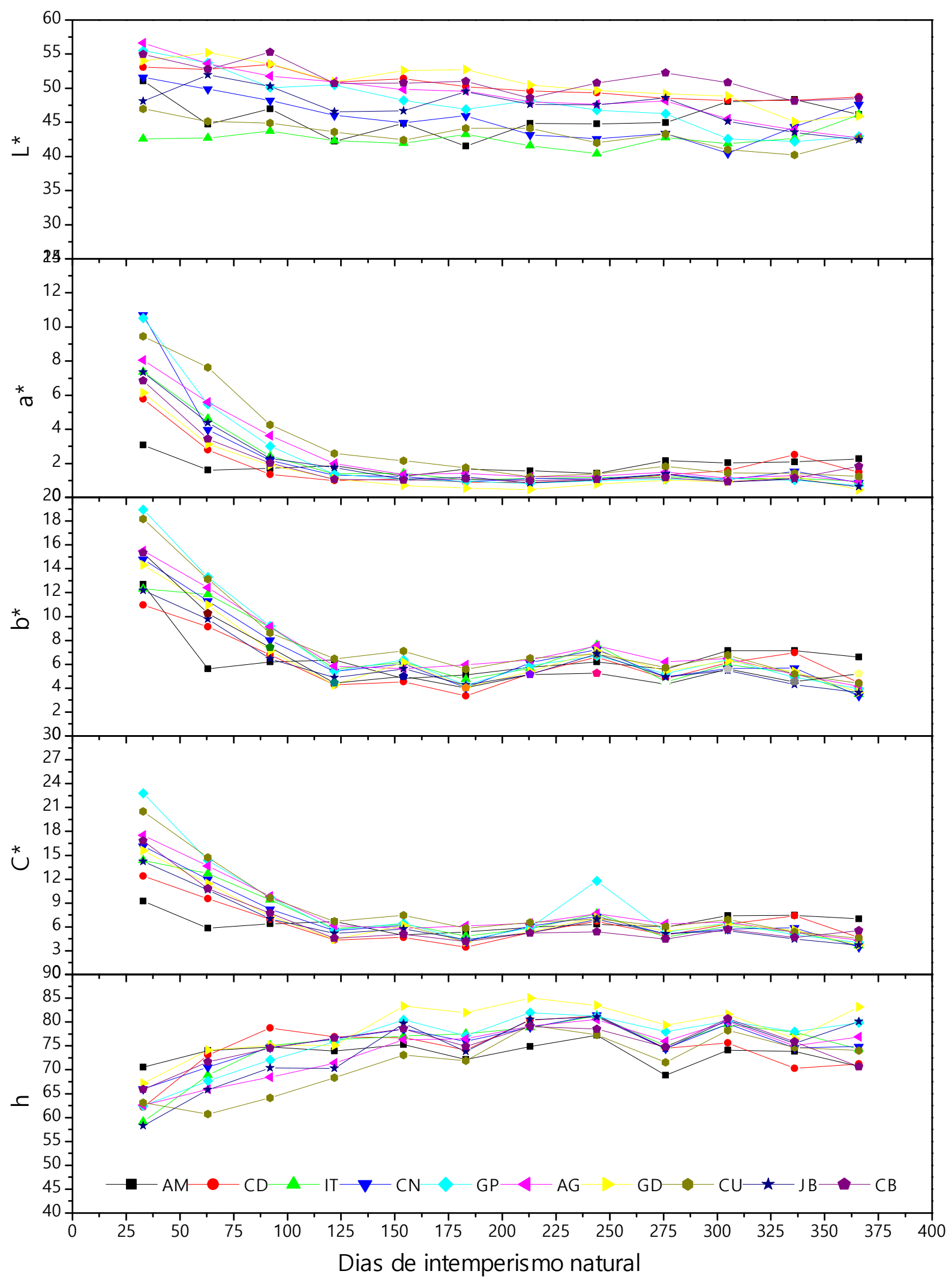

Figura 2. Parâmetros colorimétricos das madeiras de dez espécies amazônicas expostas ao intemperismo natural. (em que: AM $=$ Amescla; $\mathrm{AG}=$ Angelim; $\mathrm{CB}=$ Cambará; $\mathrm{CN}=$ Canelão; $\mathrm{CD}=$ Cedro CU = Cumaru; GD = Guanandi; GP - Garapeira; IT = Itaúba; JB = Jatobá).

Figure 2. Colorimetric paramters of tem Amazonian wood species exposed to natural weathering (in which: $\mathrm{AM}=\mathrm{Amescla}$; $\mathrm{AG}=$ Angelim; $\mathrm{CB}=$ Cambará; $\mathrm{CN}$ = Canelão; CD = Cedro; CU = Cumaru; GD = Guanandi; GP - Garapeira; IT = Itaúba; JB = Jatobá). 
$\mathrm{O}$ parâmetro $\mathrm{h}$ (ângulo de tinta) apresentou comportamento inverso ao das demais coordenadas colorimétricas, com exceção para a madeira de Amescla. As menores variações crescentes ao longo do tempo para o ângulo de tinta (h) foram observadas na madeira de Canelão. Martins et al. (2011) e Cademartori et al. (2015) também verificaram acréscimo nos valores do parâmetro $h$ ao avaliarem madeiras do gênero Eucalyptus expostas aos ensaios de intemperismo artificial e natural, respectivamente.

No geral, as madeiras que apresentaram maiores variações totais da cor $(\bullet E)$ foram a Amescla $(34,69)$ e Angelim $(31,12)$, enquanto, a madeira do Cedro teve menor - E $(17,48)$. A Figura 3 mostra o quanto a exposição natural afetou a coloração das madeiras estudadas, podendo ser um indicativo, de que, quando em uso, recomenda-se o tratamento superficial para as espécies que apresentam maior - E.

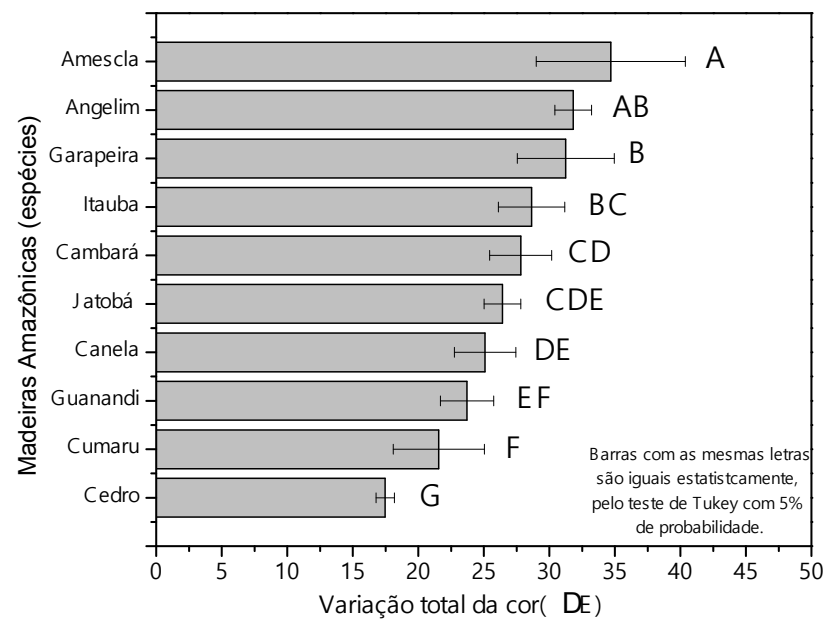

Figura 3. Variação total da cor $(\bullet E)$ das madeiras de dez espécies amazônicas expostas ao intemperismo natural.

Figure 3. Total color variation $(\bullet$ E) of ten Amazonian wood species exposed to natural weathering.

Visando analisar de forma mais detalhada a variação de cor das madeiras, empregou-se a análise de componentes principais (ACP) para agrupamento das espécies (Figura 4), como uma forma de gerar informações da cor da madeira expressa pelo conjunto e não individualmente. Esse tipo de análise, baseia-se na estatística multivariada, pois em geral, os fenômenos ambientais dependem de muitas variáveis (como nesse caso da variação colorimétrica da madeira), com isso não basta conhecer as variáveis isoladas, mas conhecê-las na sua totalidade, pois uma depende da outra e as informações são fornecidas pelo conjunto e não individualmente (MINGOTI, 2005; LOBÃO et al., 2011; HONGYU et al., 2015).

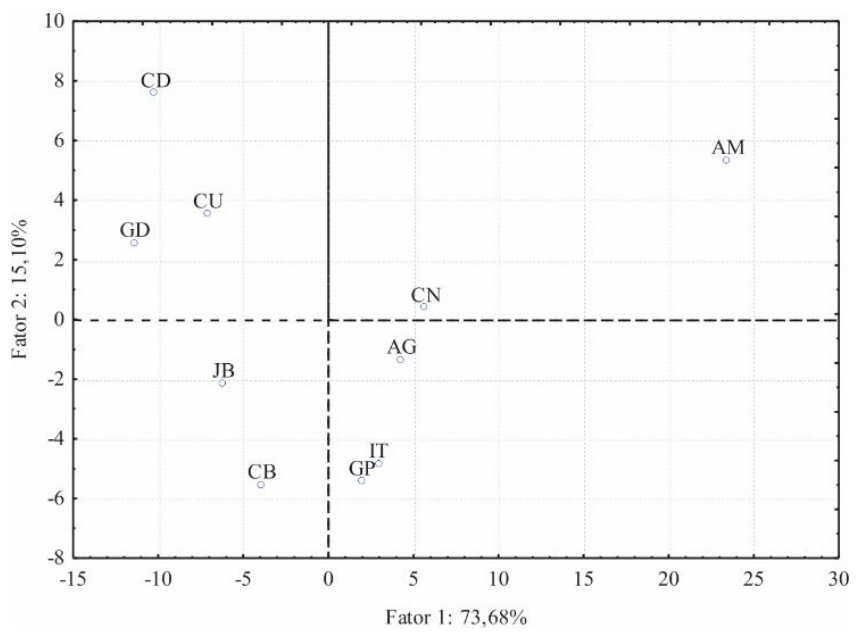

Figura 4. Análise de componentes principais para os parâmetros colorimétricos iniciais $\left(L^{*}, a^{*}, b^{*}, C^{*} e h\right)$, separados por semelhança de cores, para madeiras de dez espécies amazônicas submetidas ao intemperismo natural. (em que: $\mathrm{AM}=$ Amescla; $\mathrm{AG}=$ Angelim; $\mathrm{CB}=$ Cambará; $\mathrm{CN}$ = Canelão; $\mathrm{CD}=$ Cedro; $\mathrm{CU}=$ Cumaru; $\mathrm{GD}=$ Guanandi; $\mathrm{GP}$ - Garapeira; IT = Itaúba; JB = Jatobá).

Figure 4. Principal component analysis for the initial colorimetric parameters $\left(\mathrm{L}^{*}, \mathrm{a}^{*}, \mathrm{~b}^{*}, \mathrm{C}^{*}\right.$ and $\mathrm{h}$ ) divided by color similarity of ten Amazonian wood species subjected to natural weathering (in which: $\mathrm{AM}=$ Amescla; $\mathrm{AG}=$ Angelim; $\mathrm{CB}$ = Cambará; $\mathrm{CN}$ = Canelão; $\mathrm{CD}=$ Cedro; $\mathrm{CU}=$ Cumaru; $\mathrm{GD}=$ Guanandi; GP - Garapeira; IT = Itaúba; JB = Jatobá).

Foram considerados os dois primeiros componentes (Fator 1 e Fator 2), pois estes conseguem reter, cumulativamente, a quantidade suficiente da informação total contida no conjunto das variáveis originais $\left(\mathrm{L}^{*}, \mathrm{a}^{*}, \mathrm{~b}^{*}, \mathrm{C}\right.$ e h). Constatou-se que o Fator 1 e Fator 2 armazenaram $88,83 \%$ da variância original dos parâmetros colorimétricos 
das madeiras. Nesse sentido, as espécies encontradas no mesmo quadrante são consideradas como pertencente ao mesmo grupo colorimétrico.

Pela distribuição das espécies na ACP (Figura 5), é possível notar a grande distinção da coloração inicial da madeira de Amescla em relação as demais espécies avaliadas. Na ACP realizada a após a exposição das madeiras ao intemperismo natural (360 dias), os Fatores 1 e 2 explicaram 95,91\% da variância das condições finais de cores das madeiras, indicando que as cores apresentaram maior similaridade quando comparado as condições iniciais. As mudanças das posições das madeiras nos quadrantes, em relação as condições iniciais, mostram que, após o ensaio de campo (intemperismo natural), algumas espécies apresentam semelhanças visuais e nos parâmetros da cor.

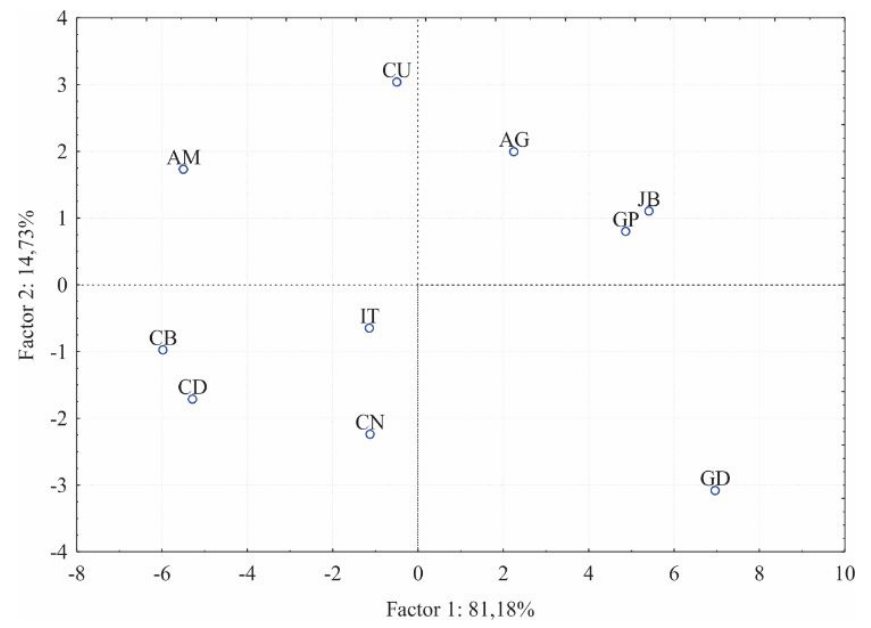

Figura 5. Análise de componentes principais para os parâmetros colorimétricos finais $\left(L^{*}, a^{*}, b^{*}, C^{*} e h\right)$, separados por semelhança de cores, para madeiras de dez espécies amazônicas submetidas ao intemperismo natural. (em que: $\mathrm{AM}=$ Amescla; $\mathrm{AG}=$ Angelim; $\mathrm{CB}=$ Cambará; $\mathrm{CN}$ = Canelão; $\mathrm{CD}=$ Cedro; $\mathrm{CU}=$ Cumaru; $\mathrm{GD}=$ Guanandi; $\mathrm{GP}$ - Garapeira; IT = Itaúba; JB = Jatobá).

Figure 5. Principal component analysis for the final colorimetric parameters $\left(\mathrm{L}^{*}, \mathrm{a}^{\star}, \mathrm{b}^{\star}, \mathrm{C}^{\star}\right.$ and $\mathrm{h}$ ) divided by color similarity of ten Amazonian wood species subjected to natural weathering (in which: $A M=$ Amescla; $A G=$ Angelim; $\mathrm{CB}=$ Cambará; $\mathrm{CN}=$ Canelão; $\mathrm{CD}=$ Cedro; $\mathrm{CU}=$ Cumaru; GD = Guanandi; GP - Garapeira; IT = Itaúba; JB = Jatobá).

\section{Conclusões}

O teor de umidade de equilíbrio (TUE) de madeiras amazônicas expostas em ensaios de campo (intemperismo natural) sofre influência da densidade básica $(\mathrm{Db})$ e da estação hídrica regional, com menores variações mensais de TUE para espécies com menores Db e na estação seca (maio a setembro).

Os parâmetros colorimétricos reduziram ao longo do tempo de exposição (exceto o ângulo de tinta). As madeiras com menores $\mathrm{Db}$ apresentaram maiores reduções do parâmetro $\mathrm{L}^{\star}$. O comportamento das coordenadas cromáticas $b^{*} e a^{*}$ indica que as madeiras perderam as tonalidades amarelas e vermelhas da caracterização inicial.

As madeiras de cedro, cumaru e guanandi apresentam menores variações totais da cor ao longo do tempo em ensaios de campo.

\section{Referências}

ABNT - ASSOCIAÇÃO BRASILEIRA DE NORMAS TÉCNICAS. NBR 7190: Projetos de estruturas de madeira. Rio de Janeiro. 1997. 107p.

ASTM - American Society for Testing and Materials. D 2244: Standard practice for calculation of color tolerances and color differences from instrumentally measured color coordinates. ASTM International: West Conshohocken. 2009. 11p.

BONFATTI JÚNIOR, E. A.; LENGOWSKI, E. C. Colorimetria aplicada à ciência e tecnologia da madeira. Pesquisa Florestal Brasileira, Colombo, v.38, e201601394, p.1-13, 2018.

CADEMARTORI, P. H. G.; MISSIO, A. L.; MATTOS, B. D.; GATTO, D. A. Natural weathering performance of three fastgrowing eucalypt woods. Maderas. Ciencia y Tecnologia, v.17, n.4, p.799-808, 2015.

CAMARgOS, J. A. A.; GONÇALEZ, J. C. A colorimetria aplicada como instrumento na elaboração de uma tabela de cores de madeira. Brasil Florestal, v.20, n.71, p.30-41, 2001. 
CASSIANO, C.; SOUZA, A. P.; STANGERLIN, D. M.; PAULINO, J.; MELO, R. R. Sazonalidade e estimativas da umidade de equilíbrio de madeiras amazônicas em Sinop, Estado do Mato Grosso. Scientia Forestalis, Piracicaba, v.41, n.100, p.457-468, 2013.

FAO. Global forest resources assessment. Food and Agriculture Organization of the United Nations, Rome, 2015. 36 .

GLASS, S. V.; ZELINKA, A. L. Moisture relations and physical properties of wood. General Technical Report FPLGTR. 2010. 190:20 pp.

HAIR JUNIOR, J. F.; BLACK, W. C.; BABIN, B. J.; ANDERSON, R. E.; TATHAM, R. L. Análise multivariada de dados. 6 ed. Porto Alegre: Bookman, 2009. 688p.

HONGYU, K.; SANDANIELO, V. L. M.; OLIVEIR JUNIOR, G. J. de. Análise de componentes principais: resumo teórico, aplicação e interpretação. Engineering and Science, Cuiabá, v. 5, n. 1, p. 83-90, 2015.

HON, D. N. S. Wethering and photochemistry of wood. In: HON, D. N. S.; SHIRAISHI, N. (Ed.). Wood and cellulosic chemistry. New York, NY: Marcel Dekker Inc., 2001. p. 512546.

JANKOWSKY, I. P.; GALVÃO, A. P. M. Influência do teor de extrativos na umidade de equilíbrio da madeira. IPEF, Piracicaba, v.18, n.1, p.1-33, 1979.

JIS_Japanese Industrial Standard. B 0601: Geometrical Products Specifications (GPS) - Surface texture: Profile method - Terms, definitions and surface texture parameters. JIS: Tokyo. 2001. 27p.

KERBER, P. R.; STANGERLIN, D. M.; PARIZ, E.; MELO, R. R. SOUZA, A. P.; CALEGARI, L. Colorimetry and surface roughness of three amazon woods submitted to natural weathering. Nativa, Sinop, v.4, n.5, p.303-307, 2016.

KILIC, A.; NIEMZ, P. Extractives in some tropical woods. European Journal of Wood and Wood Products, v.70, n.1, p.79-83, 2012.

LOBÃO, M. S.; CASTRO, V. R. de; RANGEL, A.; SARTO, C.; TOMAZELLO FILHO, M.; SILVA JÚNIOR, F. G. da; CAMARGO NETO, L. de; BERMUdEZ, M. A. R. C. Agrupamento de espécies florestais por análises univariadas e multivariadas das características anatômica, física e química das suas madeiras. Scientia Forestalis, Piracicaba, v. 39, p. 469-477, 2011.
MADY, F. T. M. Conhecendo a madeira: informações sobre 90 espécies comerciais. Manaus: SEBRAE/AM/Programa de Desenvolvimento Empresarial e Tecnológico, 2000. 212 p.

MARTINS, S.; SANTOS, C. M. T.; GONÇALEZ, J. C.; CAMARGOS, J. A. A. Envelhecimento artificial acelerado por radiação ultravioleta de madeiras de Eucalyptus benthamii e Pinus caribaea var. hondurensis. Floresta, Curitiba, v. 41, n. 1, p. 87-96, 2011.

MARTINS, V. A.; ALVES, M. V. S.; SILVA, J. F.; REBELLO, E. R. G.; PINHO, G. S. C. Umidade de equilíbrio e risco de apodrecimento da madeira em condições de serviço no Brasil. Brasil Florestal, v. 76, n. 1, p. 29-34, 2003.

MELO, J. E.; CORADIN, V. T. R.; MENDES, J. C. Classes de densidade para madeiras da Amazônia brasileira. In: Congresso Florestal Brasileiro, VI, Campos do Jordão. Anais... São Paulo: SBS/SBSF, p.695-699, 1990.

MINGOTI, S.A. Análise de dados através de métodos de estatística multivariada: uma abordagem aplicada. Belo Horizonte: Ed. UFMG, 2005. 297p.

OLIVEIRA, J. T. S. Estudos das propriedades físicas e tecnológicas da madeira de pindaíba (Xylopia sericea St. Hill.). Viçosa: Universidade Federal de Viçosa, 1988. $106 f$. Dissertação de Mestrado.

OZYHAR, T.; HERING, S.; NIEMZ, P. Moisture-dependent elastic and strength anisotropy of European beech wood in tension. Journal of Materials Science, v.47, n.16, p.61416150, 2012.

QUINTILHAN, M. T.; OLIVEIRA, W. C. de; OLIVEIRA, A. C.; PEREIRA, B. L. C.; MÔRA, R.; PINTO, A. A. de S. Deterioração da madeira de Eucalypyus e Corymbia em ensaio de campo. Ciência da Madeira, Pelotas, v.9, n.2, p.8294, 2018.

RIBEIRO, D. S.; GONÇALVES, A. L.; MELO, C. F.; REIS, A. R. S. Reação da densidade e das propriedades mecânicas de três espécies amazônicas submetidas a ensaio de campo. Ciência da Madeira, Pelotas, v.10, n.1, p.18-28, 2019.

RIBEIRO, M. A.; STANGERLIN, D. M.; SOUZA, A. P. de; CARDOSO, G. V.; CALERIGARI, L.; GATTO, D. A. Durabilidade natural da madeira de jequitibá em ensaios de deterioração em campo aberto e floresta durante as estações de seca chuva. Comunicata Scientiae, Bom Jesus, v.5, n.4, p.402-411, 2014. 
ROMANINI, A.; STANGERLIN, D. M.; PARIZ, E.; SOUZA, A. P. de; GATTO, D. A.; CALEGARI, L. Durabilidade natural da madeira de quatro espécies amazônicas em ensaios de deterioração de campo. Nativa, Sinop, v.2, n.1, p.13-17, 2014

ROWEL, R.M. Wood chemistry and wood composites. Boca Raton: CRC Press, 2005. 473p.

SANTOS, R. B.; SOUZA, A. P.; SILVA, A. C.; ALMEIDA, F. T.; ARANTES, K. R.; SIQUEIRA, J. L. Planejamento da pulverização de fungicidas em função das variáveis meteorológicas na região de Sinop-MT. Global Science and Technology, Rio Verde, v.6, n.1, p.72-88, 2013.

SILVA, R. A. F. da; SETTER, C.; MAZETTE, S. S.; MELO, R. R. de; STANGERLIN, D. M. Colorimetria da madeira de trinta espécies tropicais. Ciência da Madeira, Pelotas, v.8, n.1, p.36-41, 2017.

SILVA, J. O.; PASTORE, T. C. M. Fotodecomposição e proteção de madeiras tropicais. Floresta e Ambiente, Seropédica, v.11, n.2, p.17-23, 2004.

SILVEIRA, L. H. C.; REZENDE, A. V.; DO VALE, A. T. Teor de umidade e densidade básica da madeira de nove espécies comerciais amazônicas. Acta Amazônica, Manaus, v. 43, n. 2, p. 179-184, 2013

SOUZA, A. P.; MOTA, L. L.; ZAMADEI, T.; MARTIM, C. C.; ALMEIDA, F. T.; PAULINO, J. Classificação climática e balanço hídrico climatológico no estado de mato grosso. Nativa, Sinop, v.1, n.1, p.34-43, 2013.

STANGELIN, D. M.; COSTA, A. F. da; GONÇALVES, J. C.; PASTORE, T. C. M.; GARLET, A. Monitoramento da biodeterioração da madeira de três espécies amazônicas pela técnica da colorimetria. Acta Amazônica, Manaus, v.43, n.4, p.429-438, 2013.

STERNADT, G. H.; CAMARGOS, J. A. A. Ação da luz solar sobre 62 espécies de madeiras da Região Amazônica. Brasília: IBAMA. 1991. 14p.

TURKOGLU, T.; BAYSAL, E.; TOKER, H. The effects of natural weathering on color stability of impregnated and varnished wood materials. Advances in Materials Science and Engineering, v.15, n.1, p.1-9, 2015.

VAREJÃO-SILVA, M. A. Meteorologia e climatologia. Brasília: INMET, 2005. 552p.
ZERBINI, N. J. Madeiras tropicais com potencial comercial da região do rio Xingu (Pará, Brasil): propriedades tecnológicas e cadeia produtiva. Brasília: Universidade de Brasília, 2008. 212f. Tese de Doutorado. 